\title{
Patients' Radiation Shielding in Interventional Radiology Settings: A Systematic Review
}

\author{
Mohamed T. El-Diasty ${ }^{1}$, Ahmed A. Olfat ${ }^{2}$, Ahmad S. Mufti ${ }^{2}$, Ahmed R. Alqurashi ${ }^{1}$, Mohammed J. \\ Alghamdi ${ }^{1}$ \\ 1. Department of Radiology, King Abdulaziz University Hospital, Jeddah, SAU 2. Department of Radiology, King \\ Abdullah Medical City, Mecca, SAU
}

Corresponding author: Ahmed A. Olfat, aao92@hotmail.co.uk

\begin{abstract}
As a result of the increasing risk of developing radiation-related complications, many approaches aimed at reducing this risk and enhancing the outcomes of the patient, doctor or device operator have been developed. In this systematic review, we aim to discuss previous investigations that studied patient shielding or protection within the context of selected interventional radiology procedures. We included original studies that used $\mathrm{K}_{\mathrm{a}, \mathrm{r}}$, and $\mathrm{P}_{\mathrm{KA}}$ for the assessment of the outcomes of two procedures: transjugular intrahepatic portosystemic shunt creation (TIPS) and hepatic arterial chemoembolization (HAE). A thorough search strategy was conducted on relevant databases to identify all relevant studies. We included 13 investigations, including 12 cross-sectional studies and one randomized controlled trial. Significant diversity was found among all these studies in terms of the used modalities, which made them hard to compare. However, almost all studies agreed that using novel imaging and interventional modalities is useful when obtaining better outcomes and reducing patient radiation exposure. The use of ultrasoundguided procedures and providing adequate lead curtains has also been recommended by the identified studies in order to minimize the frequency of radiation exposure. The reported $\mathrm{K}_{\mathrm{a}, \mathrm{r}}$, and $\mathrm{P}_{\mathrm{KA}}$ were also variable between studies and were discussed within this study. Our findings indicate that unified guidelines for patient radiation shielding should be urgently investigated.
\end{abstract}

Categories: Preventive Medicine, Radiation Oncology, Radiology

Keywords: interventional, radiology, transjugular intrahepatic portosystemic shunt, hepatic arterial chemoembolization, shielding

\section{Introduction And Background}

As a result of increasing medical advances in interventional radiology techniques and the reported benefits of various related fluoroscopic guided procedures, there can be prolonged procedures exposing the patient and staff to higher amounts of radiation with potential short- and long-term effects. However, this has been met by concern and caution as the increased prevalence of these procedures subsequently increases the risks associated with radiation exposure. Although it is now clear that interventional radiology plays an important role in the management of many diseases and fewer reported complications than those resulting from invasive surgical procedures, previous studies have demonstrated that interventional radiological procedures may be associated with increased side effects including hair loss and other tissue-reactive abnormalities that are secondary to the increased exposure to radiation [1-5].

El-Diasty et al. This is an open access article distributed under the terms of the Creative Commons Attribution License CC-BY 4.0., which permits unrestricted use, distribution, and reproduction in any medium, provided the original author and source are credited.

There are many precautions and steps that can protect against complications related to interventional radiological procedures. In particular, which require the integration of novel modalities to reduce the maximum skin dose during radiation exposure (Dskin, max) [6,7]. Additionally, previous studies have demonstrated that an estimation of Dskin, max can be undertaken using various modalities including metaloxide-semiconductor field-effect transistors, four photoluminescence sensors attached to the back of the patients, and wireless dosimeter modalities [8-11]. Although this approach has been previously reported to have various benefits when estimating the real-time data of radiation exposure, subsequent evidence indicates that it had some limitations which restrict its benefits to specific institutions [12,13]. As a result of these limitations, previous investigations reported the efficacy of both air kerma at the patient entrance reference point (Ka,r), and the directly assessed Dskin, max of the exposed patients [14-16]. Moreover, it has been indicated that both modalities have a significant correlating factor, and estimating the value of any of these modalities can obtain that of the other [17-19].

As a result of the increased risk of developing radiation-related complications, many suggestions have been made to reduce the risk and enhance the outcomes for the patient, doctor or device operator. Some studies reviewed here reported a potential reduction of radiation risk in their population as measured by Ka,r, and air kerma-area product (PKA) [20]. As such, in this systematic review we aim to address those studies that have assessed patient shielding or protection in selected interventional radiology procedures, as measured by the aforementioned two parameters. 


\section{Review \\ Methods}

Definition of Outcomes and Inclusion Criteria

In this systematic review, we aim to discuss those previous investigations that have studied patient shielding or protection in the setting of selected interventional radiology procedures. We have included original studies that encompass hepatic-related procedures as these were commonly found in the literature with sufficient population and reporting of outcomes. Moreover, it has been identified that these procedures imply that patients are more frequently exposed to irradiation than other specialized interventional procedures. Accordingly, we only included studies that were based on creating transjugular intrahepatic portosystemic shunt (TIPS) and hepatic arterial chemoembolization (HAE) procedures.

Studies that used the $\mathrm{K}_{\mathrm{a}, \mathrm{r}}$, and $\mathrm{P}_{\mathrm{KA}}$ were utilized for the assessment of these outcomes. $\mathrm{K}_{\mathrm{a}, \mathrm{r}}$ usually refers to the initial kinetic energy that was released per total mass of the released air per the interventional reference point. This has been previously determined by the International Electrotechnical Commission to be 60601-243, and is measured by Gy. The location of the Ka,r has been previously referred to as the center of the X-ray bundle and $15 \mathrm{~cm}$ away from the $\mathrm{C}$-arm isocenter of the same bundle, which is usually located within or towards the focal spot. In clinical settings, Ka,r can be used to assess whether the threshold of radiation skin exposure has been exceeded, which can prevent the development of complications. It can also classify the severity of the clinical outcomes based on the dose of radiation. Previous studies have demonstrated that when excluding fluoroscopically guided interventions, Ka,r is the best modality to assess skin radiation exposure. Conversely, PKA or dose area product (DAP) is measured by Gy per $\mathrm{cm}^{2}$ and usually refers to the plane where the area of the X-ray beam and the cross-sectional area of the Ka,r has been identified. In clinical settings, PKA can be used for the estimation of stochastic risk as it can estimate the total effective dose of radiation and the total amount of energy consumed. Stochastic effects are important and can cause serious complications and affect the patient's quality of care. All of the included articles in this review have assessed their outcomes on a patient population.

Articles that did not assess the aforementioned outcomes or identified procedures were excluded. Articles that were not original or did not investigate the outcomes or the effect of dose reduction on a patient population were also excluded, as were theses, protocols, abstract-only articles, and non-English studies.

\section{Search Strategy}

Our search strategy was carefully designed to identify all related citations, and the study members also identified relevant articles to assist with locating all relevant databases. The search terms were modified when needed. The search strategy was confined to articles that were published post-2011 and any articles published before this period were excluded. We searched PubMed, Scopus, Google Scholar, Embase, Cochrane library, Virtual Health Library, and Web of Science. Review articles were also used to identify any relevant citations that could have been missed by the main electronic search strategy.

\section{Article Screening}

This step was undertaken by all study authors, which required the integration of all efforts to obtain the best outcomes. When the search was complete, the study leader exported all the results, which had been agreed to by all members, to a unified endnote library designed to exclude the duplicated studies identified by the different database searches. The remaining articles were then exported into an excel datasheet for criteriabased screening. Title and abstract screening were followed by full-text downloading and each screening was undertaken by at least two researchers for each article, and each of these researchers was blind to the results of the other to restrict cheating and collusion. Discussions between the researchers and supervision by a senior leader were also utilized whenever a conflict was observed among the screened articles. All steps were undertaken per the Preferred Reporting Items for Systematic Reviews and Meta-Analyses (PRISMA) guidelines [21].

Data Extraction and Quality Assessment

After the final check of the screened articles, a suitable extraction spreadsheet was created to contain all the relevant information that could help us report our outcomes. The spreadsheet consisted of three parts. First part included a section for baseline characteristics such as author name, country, DOI, sample size, study design, gender, and age. The second part consisted of the outcomes such as the type of the procedure, the applied radiation interventional modality, and the estimated $\mathrm{P}_{\mathrm{KA}}$, DAP, and $\mathrm{K}_{\mathrm{a}, \mathrm{r}}$. Lastly, the last spreadsheet contained the quality assessment. This section was conducted using the modified Newcastle-Ottawa scale (NOS) for cross-sectional studies [22]. The scale is composed of three main domains assessing the quality of assessment, reporting, and compatibility. Studies were classified based on their assessed grades and quality standards. 


\section{Cureus}

\section{Results}

Study Selection

A summary of this step can be seen in Figure 1 flow chart. In brief, we exported a total of 6,655 citations to the endnote library, which was then used to exclude duplicates. The final screening of the remaining articles identified 13 related studies that met our criteria.

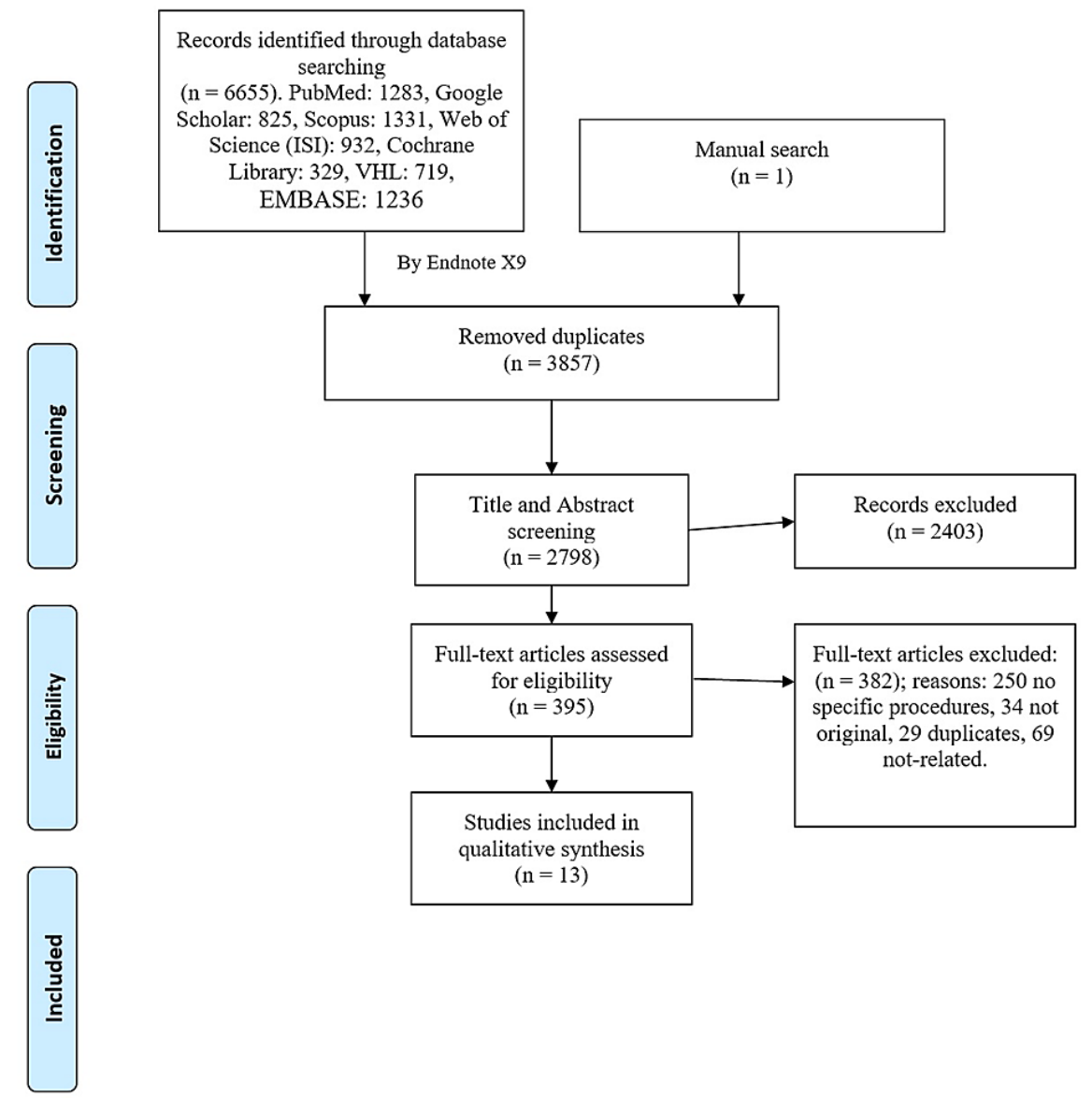

FIGURE 1: PRISMA flow chart for the selection process to include the relevant studies.

PRISMA: Preferred Reporting Items for Systematic Reviews and Meta-Analyses.

Risk of Bias

None of the included studies in this review had a non-satisfactory quality of bias as assessed by our authors. All studies showed favorable qualities as the results ranged between good and satisfactory markings according to the grades of these studies (graded from 0 to 10). The specific themes and grading of domains of the web of science (WOS) scale are presented in Table 1. Only one randomized controlled trial was included and our assessment indicated that the study had a low risk of bias according to Cochrane's collaboration tool for assessment of bias in randomized studies. 


\section{Cureus}

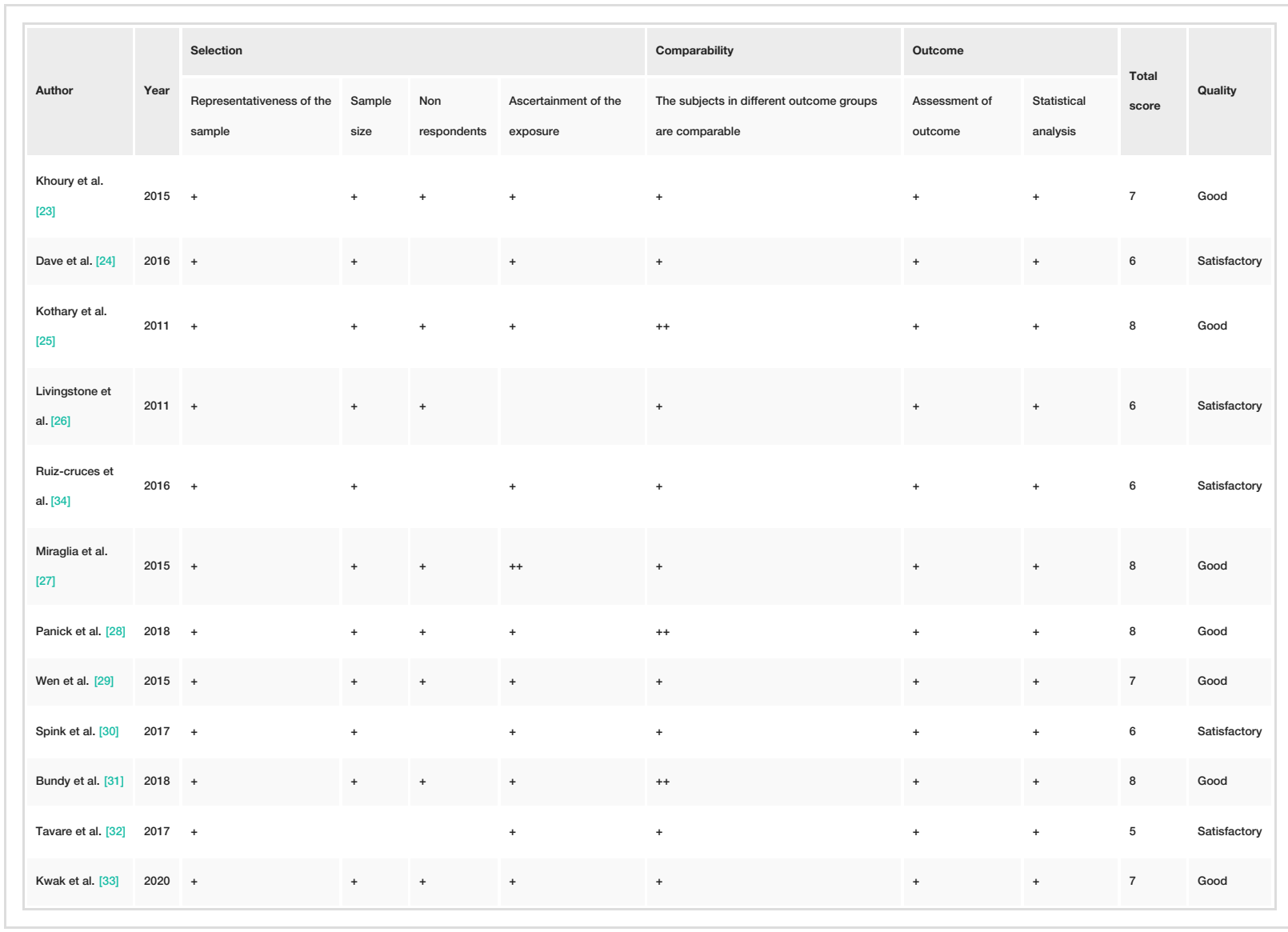

TABLE 1: Quality assessment of the included studies by the modified Newcastle-Ottawa scale (NOS).

Baseline Characteristics

This systematic review was comprised of a total of 12 relevant cross-sectional studies and one randomized controlled trial. All articles contributed to the investigation of our declared outcomes and helped us assess our final evidence. Among these studies, six were from the United States, and one each from Brazil, India, China, Italy, Germany, Spain, and the United Kingdom. All of the studies were prospective bar two studies that were based on a retrospective analysis. The number of performed procedures in the interventional radiology units was also variable, ranging between 11 and 212 procedures. Moreover, six studies included patients that underwent TIPS procedures only, another six included patients who underwent only HAE procedures, and only one study included both procedures in their population. Other baseline characteristics and a summary of the outcomes can be seen in Table 2 . 


\section{Cureus}

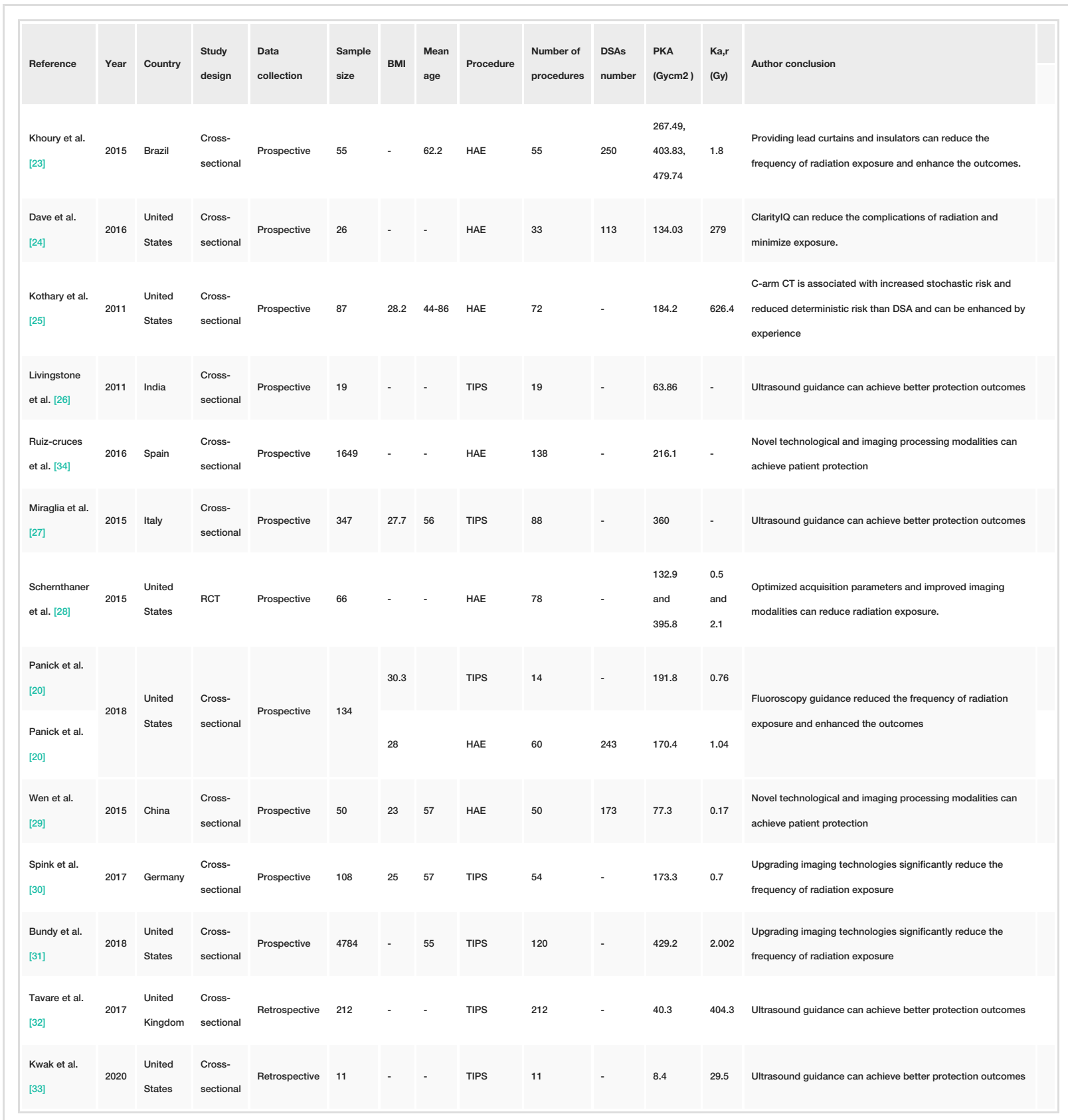

TABLE 2: Baseline characteristics and summary of the outcomes of the included studies.

\section{Discussion}

TIPS

Many of the studies included in this review assessed the efficacy of their novel approaches for reducing the dose of radiation, minimizing the risk of developing radiation exposure, and achieving patient protection. The investigation by Livingstone et al. [26] reported that significant radiation protection was obtained in their population after the introduction of ultrasound-guided modalities for their patients resulted in lower estimated total DAP levels. This was also supported by the Miraglia et al. [27], Tavare et al. [32], and Zhang et al. [33] studies, which reported that DAP levels were significantly lower in patients who underwent ultrasound-guided procedures. A previous investigation by Panick et al. [20] also evaluated the efficacy of a new modality (Discovery IGS740, GE Healthcare) for obtaining better outcomes in multiple hepatic procedures. The authors reported a significant reduction in both the $\mathrm{P}_{\mathrm{KA}}$ and $\mathrm{K}_{\mathrm{a}, \mathrm{r}}$ levels for TIPS procedures, as noticed in the novel room when compared to the other previous modalities. The AlluraClarity was also developed by Spink et al. [30] in 2017 and demonstrated a significant reduction in radiation parameters without interfering with the quality of the outcomes. Bundy et al. [31] also compared their approach to the RAD-IR study conducted in 2003 and reported that the previously proposed reference levels for DAP and 
time spent on fluoroscopy were identified among their recommended procedures, including TIPS and hepatic chemoembolization.

$H A E$

The effect of patient protection and radiation dose reduction in HAE procedures was also investigated by many of the selected articles. The previous study by Khoury et al. [23] reported the radiation doses for patients treated across three hospitals. They reported differences between the total $\mathrm{P}_{\mathrm{KA}}$ levels $(267.49$,

403.83, and $479.74 \mathrm{~Gy} \mathrm{~cm}^{2}$ ) between the three hospitals, who each used a different imaging modality. Accordingly, the authors suggested that interventional approaches such as lead shields should be adequately installed in the hospitals to avoid possible skin-related complications. However, these were not widely available in all healthcare settings, and when available, they were not widely used by the attending physicians. Another study by Dave et al. [24] reported that the $\mathrm{P}_{\mathrm{KA}}$ and $\mathrm{K}_{\mathrm{a}, \mathrm{r}}$ levels were significantly lower when the new imaging modality Clarity IQ was installed in their settings, however, the imaging quality for this technique was relatively lower than other modalities that did not feature the technique. The study by Kothary et al. [25] reported that the application of a C-arm CT was associated with more frequent stochastic risk, as expressed by DAP, and increased reduction in the deterministic risk than the digital subtraction angiography procedure. However, they also reported that the quality of protection with the C-arm modality can be enhanced by increasingly experienced physicians. Positive instances of $\mathrm{C}$-arm imaging technique use were reported by Schernthaner et al. [28], who demonstrated that the modality was able to reduce the time of the procedure without interfering with the quality of the obtained images. The Ruiz-Cruces et al. [34] study stratified the diagnostic reference levels for hepatic chemoembolization procedures based on the complexity of the procedure. They reported that the $\mathrm{P}_{\mathrm{KA}}$ levels for the simple, medium and complex procedures should

not exceed 170, 303, and $881 \mathrm{~Gy} \mathrm{~cm}^{2}$. Therefore, the complexity of the procedure must be estimated in order to determine the optimum dose of radiation. The new suite developed by Panick et al. [20] also managed to obtain better outcomes and protection in HAE procedures when compared to previously studied modalities. Similarly, a new system was also developed by Wen et al. [29] which demonstrated less radiation exposure with a maintained imaging quality, as a result of the reduced time and energy of exposure.

Our results may be limited to the designs of the included studies, which lacked proper randomization of patients and adequate sample sizes. Moreover, we could not formulate adequate analyses to obtain the most favorable and efficacious modalities for reducing exposure to radiation during radiological interventions, due to the heterogeneity among the included studies.

\section{Conclusions}

Our systematic review discussed current approaches to achieving patient radiation protection. We found great diversity among the included studies and almost all developed a novel strategy for achieving patient protection. However, most studies agreed on using combination of imaging-processing techniques and ultrasound-guided interventions for enhancing outcomes, thus reducing radiation exposure and decreasing the frequency of potential complications. Unified guidelines for these processes should be urgently considered via further investigations.

\section{Additional Information \\ Disclosures}

Conflicts of interest: In compliance with the ICMJE uniform disclosure form, all authors declare the following: Payment/services info: All authors have declared that no financial support was received from any organization for the submitted work. Financial relationships: All authors have declared that they have no financial relationships at present or within the previous three years with any organizations that might have an interest in the submitted work. Other relationships: All authors have declared that there are no other relationships or activities that could appear to have influenced the submitted work.

\section{References}

1. Wagner LK, Eifel PJ, Geise RA: Potential biological effects following high X-ray dose interventional procedures. JVIR. 1994, 5:71-84. 10.1016/s1051-0443(94)71456-1

2. Mooney RB, McKinstry CS, Kamel HA: Absorbed dose and deterministic effects to patients from interventional neuroradiology. Br J Radiol. 2000, 73:745-51. 10.1259/bjr.73.871.11089467

3. Hayakawa M, Moritake T, Kataoka F, et al.: Direct measurement of patient's entrance skin dose during neurointerventional procedure to avoid further radiation-induced skin injuries. Clin Neurol Neurosurg. 2010, 112:530-6. 10.1016/j.clineuro.2010.03.019

4. Magrassi L, Bongetta D, D'Ercole L, Lisciandro F, Arienta C, Thyrion FZ: Neuroembolization may expose patients to radiation doses previously linked to tumor induction. Acta Neurochir. 2012, 154:33-41. 10.1007/s00701-011-1209-9

5. Corrigall RS, Martin CJ, Scott I: Observations of tissue reactions following neuroradiology interventional procedures. J Radiol Prot. 2020, 40:N9-N15. 10.1088/1361-6498/ab5bf4

6. Valentin J: Avoidance of radiation injuries from medical interventional procedures, ICRP Publication 85 . 
Ann ICRP. 2000, 30:7-7. 10.1016/S0146-6453(01)00004-5

7. Miller DL, Balter S, Noonan PT, Georgia JD: Minimizing radiation-induced skin injury in interventional radiology procedures. Radiology. 2002, 225:329-36. 10.1148/radiol.2252011414

8. Safari MJ, Wong JH, Kadir KA, et al.: Real-time eye lens dose monitoring during cerebral angiography procedures. Eur Radiol. 2016, 26:79-86. 10.1007/s00330-015-3818-9

9. Kato M, Chida K, Nakamura M, Toyoshima H, Terata K, Abe Y: New real-time patient radiation dosimeter for use in radiofrequency catheter ablation. J Radiat Res. 2019, 60:215-20. 10.1093/jrr/rry110

10. Inaba Y, Chida K, Murabayashi Y, Endo M, Otomo K, Zuguchi M: An initial investigation of a wireless patient radiation dosimeter for use in interventional radiology. Radiol Phys Technol. 2020, 13:321-6. 10.1007/s12194-020-00575-2

11. Sato F, Honda T, Haga Y, et al.: Basic characteristic evaluation of the real-time model MOSFET dosimeter. Bull Sch Health Sci Tohoku Univ. 2017, 26:57-65.

12. Rana VK, Rudin S, Bednarek DR: A tracking system to calculate patient skin dose in real-time during neurointerventional procedures using a biplane x-ray imaging system. Med Phys. 2016, 43:5131. $10.1118 / 1.4960368$

13. Takata T, Kotoku J, Maejima H, et al.: Fast skin dose estimation system for interventional radiology. J Radiat Res. 2018, 59:233-9. 10.1093//rr/rrx062

14. Bor D, Sancak T, Olgar T, Elcim Y, Adanali A, Sanlidilek U, Akyar S: Comparison of effective doses obtained from dose-area product and air kerma measurements in interventional radiology. Br J Radiol. 2004, 77:31522. $10.1259 / \mathrm{bjr} / 29942833$

15. Farah J, Trianni A, Ciraj-Bjelac O, et al.: Characterization of XR-RV3 GafChromic( $\left.{ }^{\circledR}\right)$ films in standard laboratory and in clinical conditions and means to evaluate uncertainties and reduce errors. Med Phys. 2015, 42:4211-26. 10.1118/1.4922132

16. Habib Geryes B, Hadid-Beurrier L, Waryn MJ, Jean-Pierre A, Farah J: Benchmarking the DACS-integrated Radiation Dose Monitor ${ }^{\circledR}$ skin dose mapping software using XR-RV3 Gafchromic ${ }^{\circledR}$ films. Med Phys. 2018, 45:4683-92. 10.1002/mp.13125

17. Nishizawa K, Moritake T, Matsumaru Y, Tsuboi K, Iwai K: Dose measurement for patients and physicians using a glass dosemeter during endovascular treatment for brain disease. Radiat Prot Dosimetry. 2003, 107:247-52. 10.1093/oxfordjournals.rpd.a006397

18. Moritake T, Matsumaru Y, Takigawa T, Nishizawa K, Matsumura A, Tsuboi K: Dose measurement on both patients and operators during neurointerventional procedures using photoluminescence glass dosimeters. AJNR Am J Neuroradiol. 2008, 29:1910-7. 10.3174/ajnr.A1235

19. Moritake T, Hayakawa M, Matsumaru $Y$, et al.: Precise mapping system of entrance skin dose during endovascular embolization for cerebral aneurysm. Radiation Measurements. 2011, 46:2103-6. 10.1016/.radmeas.2011.08.008

20. Panick C, Wunderle K, Sands M, Martin C: Patient radiation dose reduction considerations in a contemporary interventional radiology suite. Cardiovasc Intervent Radiol. 2018, 41:1925-34. 10.1007/s00270-018-2052-7

21. Liberati A, Altman DG, Tetzlaff J, et al.: The PRISMA statement for reporting systematic reviews and metaanalyses of studies that evaluate healthcare interventions: explanation and elaboration. BMJ. 2009, 339:b2700. 10.1136/bmj.b2700

22. Newcastle- Ottawa: Quality assessment scale adapted for cross-sectional studies . (Newcastle- Ottawa: Quality assessment scale adapted for cross-sectional studies). Accessed: June 2021: https://storage.googleapis.com/plos-corpus-prod/10.1371/journal.pone.0147601/1/pone.0147601.s001.pdf? X-Goog-Algorithm....

23. Khoury HJ, Garzon WJ, Andrade G, Lunelli N, Kramer R, de Barros VS, Huda A: Radiation exposure to patients and medical staff in hepatic chemoembolisation interventional procedures in Recife, Brazil. Radiat Prot Dosimetry. 2015, 165:263-7. 10.1093/rpd/ncv075

24. Dave JK, Eschelman DJ, Wasserman JR, Gonsalves CF, Gingold EL: A phantom study and a retrospective clinical analysis to investigate the impact of a new image processing technology on radiation dose and image quality during hepatic embolization. J Vasc Interv Radiol. 2016, 27:593-600. 10.1016/j.jvir.2016.01.131

25. Kothary N, Abdelmaksoud MH, Tognolini A, et al.: Imaging guidance with C-arm CT: prospective evaluation of its impact on patient radiation exposure during transhepatic arterial chemoembolization. J Vasc Interv Radiol. 2011, 22:1535-43. 10.1016/j.jvir.2011.07.008

26. Livingstone RS, Keshava SN: Technical note: Reduction of radiation dose using ultrasound guidance during transjugular intrahepatic portosystemic shunt procedure. Indian J Radiol Imaging. 2011, 21:13-4. 10.4103/0971-3026.76046

27. Miraglia R, Maruzzelli L, Cortis K, et al.: Radiation exposure in transjugular intrahepatic portosystemic shunt creation. Cardiovasc Intervent Radiol. 2016, 39:210-7. 10.1007/s00270-015-1164-6

28. Schernthaner RE, Duran R, Chapiro J, Wang Z, Geschwind JF, Lin M: A new angiographic imaging platform reduces radiation exposure for patients with liver cancer treated with transarterial chemoembolization. Eur Radiol. 2015, 25:3255-62. 10.1007/s00330-015-3717-0

29. Wen X, Jiang X, Li R, Zhang J, Yang P, Shen B: Novel X-ray imaging technology allows substantial patient radiation reduction without image quality impairment in repetitive transarterial chemoembolization for hepatocellular carcinoma. Acad Radiol. 2015, 22:1361-7. 10.1016/j.acra.2015.07.010

30. Spink C, Avanesov M, Schmidt T, et al.: Radiation dose reduction during transjugular intrahepatic portosystemic shunt implantation using a new imaging technology. Eur J Radiol. 2017, 86:284-8. 10.1016/j.ejrad.2016.11.028

31. Bundy JJ, Chick JF, Hage AN, et al.: Contemporary interventional radiology dosimetry: analysis of 4,784 discrete procedures at a single institution. J Am Coll Radiol. 2018, 15:1214-21. 10.1016/j.jacr.2018.06.004

32. Tavare AN, Wigham A, Hadjivassilou A, et al.: Use of transabdominal ultrasound-guided transjugular portal vein puncture on radiation dose in transjugular intrahepatic portosystemic shunt formation. Diagn Interv Radiol. 2017, 23:206-10. 10.5152/dir.2016.15601 


\section{Cureus}

33. Zhang WW, Ren JZ, Zhou PL, et al.: Application of real-time image fusion technique in transjugular intrahepatic portosystemic shunt. Zhonghua Yi Xue Za Zhi. 2019, 99:3554-7. 10.3760/cma.j.issn.03762491.2019.45.005

34. Ruiz-Cruces R, Vano E, Carrera-Magariño F, et al.: Diagnostic reference levels and complexity indices in interventional radiology: a national programme. Eur Radiol. 2016, 26:4268-76. 10.1007/s00330-016-4334-2 